\title{
Erratum: Pediatric kidney transplantation is different from adult kidney transplantation
}

Min Hyun Cho, MD

Department of Pediatrics, School of Medicine, Kyungpook National University, Daegu, Korea

Korean J Pediatr 2018 Jul;61(7):205-209.

https://doi.org/10.3345/kjp.2018.61.7.205.

There were errors at the sentence of 10th line in Introduction section of main text. The "(from living donors)" and "(from deceased donors)" should be removed in the sentence. The sentence should be corrected as follow.

It has been reported that at present, the 1- and 5-year patient survival rates associated with pediatric KT are approximately 98\% and 94\%, respectively, and the 1- and 5-year graft survival rates are 93\%-95\% and 77\%-85\%, respectively ${ }^{3}$ (Table 1). 